\title{
Long-Term Efficacy, Safety, and Tolerability of Modified Intravesical Oxybutynin Chloride for Neurogenic Bladder in Children
}

\author{
Masashi Honda ${ }^{\text {a c }}$, Yusuke Kimura ${ }^{\text {a }}$, Panagiota Tsounapi ${ }^{\text {a }}$, Katsuya Hikita ${ }^{\text {a }}$ \\ Motoaki Saito $^{\mathrm{b}}$, Atsushi Takenaka ${ }^{\mathrm{a}}$
}

\begin{abstract}
Background: Children with spinal cord disorders can present with neurogenic bladder, a condition in which the bladder partly or completely loses its ability to store urine and void at low pressure. A bladder with low compliance may cause urinary incontinence, which negatively impacts quality of life and renal function. Long-term high pressure neurogenic bladder can increase the risk of deterioration in renal function. Antimuscarinic pharmacotherapy with clean intermittent catheterization is currently considered one of the most effective treatments for these patients. However, some patients do not respond to oral medication or have unacceptable adverse events (AEs), which may result in medical withdrawal for these patients. Intravesical oxybutynin is an effective treatment with less AEs compared with oral medication. However, an important issue with this treatment is retention of the solution in the bladder. Moreover, as yet no data are available on the very long-term use and outcome of modified intravesical oxybutynin therapy. In the present study, we report on the efficacy, safety, and side effects of long-term modified intravesical oxybutynin therapy in children with neurogenic bladder.
\end{abstract}

Methods: Modified intravesical oxybutynin $(1.25 \mathrm{mg} / 5 \mathrm{~mL}$, twice a day) was administered to four children (three boys and one girl) with neurogenic bladder (detrusor overactivity and/or low compliance bladder), who were previously unresponsive to or experienced intolerable AEs from oral medications. Results of pretreatment cystometrograms were compared to those from follow-up urodynamic studies. Anticholinergic AEs, occurrence of urinary tract infection, and degree of incontinence during this treatment were also evaluated.

Results: After 1 year, bladder compliance improved in three of the four patients. After 3 years, detrusor overactivity was undetectable in

Manuscript submitted January 12, 2019, accepted February 25, 2019

aDepartment of Urology, Tottori University Faculty of Medicine, Yonago, Tottori 683-8504, Japan

bDepartment of Pharmacology, Kochi Medical School, KohasuOkocho, Nankoku, Kochi 783-8505, Japan

${ }^{\mathrm{c} C}$ Corresponding Author: Masashi Honda, Department of Urology, Tottori University Faculty of Medicine, 36-1 Nishi, Yonago, Tottori 683-8504, Japan.

Email: honda@tottori-u.ac.jp

doi: https://doi.org/10.14740/jocmr3752 all patients. Bladder compliance at 3 years and 10 years after initiation of therapy was similar for three patients, and they are continuing modified intravesical oxybutynin therapy. One patient discontinued therapy at 118 months due to worsening of bladder compliance and upper urinary tract infection. No anticholinergic systemic AEs were observed in any of the patients.

Conclusions: These results suggest that modified intravesical oxybutynin is an effective and relatively safe long-term therapeutic option for children with neurogenic bladder.

Keywords: Neurogenic bladder; Children; Intravesical oxybutynin chloride; Hydroxypropylcellulose

\section{Introduction}

Antimuscarinic pharmacotherapy is generally considered the gold standard treatment for patients with overactive bladder and low compliance bladder. For patients with overactive bladder and/or urinary incontinence, treatment with oral antimuscarinics is usually effective. Unfortunately, some patients do not respond to oral medications or experience unacceptable adverse events (AEs) $[1,2]$. In such patients, it has been reported that intravesical oxybutynin chloride is an effective therapy for neurogenic bladder dysfunction $[1,3$, 4], but the effects are often transient. In previous studies, we found that intravesical oxybutynin chloride solution supplemented with hydroxypropylcellulose (HPC), a mucosal adhesive substance, reduced systemic AEs, and that this therapy, which we termed modified intravesical oxybutynin therapy, was safe and showed excellent efficacy $[5,6]$. Our previous data suggest that this modification has the potential to reduce the absorption of oxybutynin from bladder mucosa so that it is retained in the bladder for longer, and that modified intravesical oxybutynin chloride may induce fewer systemic AEs than intravesical oxybutynin chloride without HPC. This treatment may also reduce unacceptable AEs in children with neurogenic bladder dysfunction. Some researchers have reported that intravesical oxybutynin chloride is effective for children with neurogenic bladder who do not respond to oral anticholinergic medication, or who experience intoler- 
Table 1. Patient Profiles

\begin{tabular}{llll}
\hline Patient & Age & Sex & Disease \\
\hline 1 & $3 \mathrm{y} 7 \mathrm{~m}$ & Male & Pelvic teratoma \\
2 & $1 \mathrm{y} 6 \mathrm{~m}$ & Male & Myelomeningocele \\
3 & $1 \mathrm{y} 6 \mathrm{~m}$ & Female & Myelomeningocele \\
4 & $2 \mathrm{y} 5 \mathrm{~m}$ & Male & Myelomeningocele \\
\hline
\end{tabular}

y: years; m: months.

able systemic AEs from these drugs [1, 7, 8]. However, there are as yet no published data on the very long-term use and outcome of modified intravesical oxybutynin therapy. In the present study, the efficacy and safety of long-term modified intravesical oxybutynin therapy in children with neurogenic bladder were examined.

\section{Materials and Methods}

\section{Patients}

The study protocol was approved by the Institutional Ethics Committee for Clinical Trials. Modified intravesical oxybutynin chloride solution supplemented with HPC was administered to four pediatric patients (three boys and one girl; all under 4 years of age) with neurogenic bladder. The patients' profiles are summarized in Table 1. Three patients (patients 2, 3, and 4) had myelomeningocele and one (patient 1) had a neurogenic bladder due to resection of a pelvic teratoma. The parents of the patients were advised about the risks and possible AEs of treatment before joining the study and signed an institutional ethics committee-approved informed consent form. All patients underwent general urological examinations as well as urodynamic studies, to verify the diagnosis of neurogenic bladder. Cohen ureterocystoneostomy was performed before modified intravesical oxybutynin chloride in patient 4 with left grade IV vesicoureteral reflux. At the time of enrollment in this study, two patients (patients 1 and 2) were being treated with oral anticholinergic medication, but this was stopped 2 weeks before the start of modified intravesical oxybutynin chloride. The other two patients (patients 3 and 4) were not receiving oral anticholinergic agents due to the pre-existence of severe constipation. Three patients (patients 1, 2, and 4) were using clean intermittent catheterization (CIC), and one patient (patient 3) was being managed with an indwelling urethral catheter because of extremely low bladder compliance.

\section{Composition of the modified oxybutynin chloride solution}

The oxybutynin chloride solution was prepared in the pharmacy division of our hospital. The solution consisted of oxybutynin chloride $2.5 \mathrm{mg}$, sodium chloride $58 \mathrm{mg}$, HPC $100 \mathrm{mg}$, sodium dihydrogenphosphate (anhydrous) $52.6 \mathrm{mg}$, disodium hydrogenphosphate (anhydrous) $8.7 \mathrm{mg}$, and water $10 \mathrm{~mL}(\mathrm{pH}$ 5.85).

\section{Evaluation of the treatment}

The modified oxybutynin chloride solution was administered according to the method described in our previous report [6]. After catheterizing to empty the bladder the modified oxybutynin chloride solution was instilled into the bladder at a dosage of $5 \mathrm{~mL}$ each time and remained until the next catheterization. This procedure was repeated twice daily. There were no changes in the frequency or dosage of oxybutynin during the course of the study. Filling cystometry was obtained before, 1 week after, and 1 year after the first instillation of modified oxybutynin chloride. Thereafter, filling cystometry was performed with the patient supine using Urodyn 5000 (Dantec, Skovlunde, Denmark) (March 2004 - March 2011) and Solar Urodynamic System (EDAP TMS, Vaulx-en-Velin, France) (April 2011 - present) every year. Sterile physiological saline solution was infused through a 7-Fr. double-lumen urethral catheter at 10 to $20 \mathrm{~mL}$ per minute with the infusion rate adjusted to patient size. Urodynamic studies were performed according to the standard methods of the International Continence Society. Cystometric bladder capacity was defined as the volume at an intravesical pressure of 30 $\mathrm{cm} \mathrm{H}_{2} \mathrm{O}$. Bladder compliance was calculated as the total volume divided by the end fill pressure. Results of pretreatment cystometrograms (CMGs) were compared to those from follow-up urodynamic studies. The patients were also carefully monitored for AEs during this treatment. In patients suspected of having a urinary tract infection (UTI), urinary samples were obtained from the catheter and cultured. All incidents of asymptomatic bacteriuria (ABU) or upper or lower UTI over the year prior to commencement of treatment and over the year following the cessation of treatment were recorded. Upper UTI was defined as a significant growth of bacteria (> $100,000 / \mathrm{mL}$ ) and a temperature $>38.5^{\circ} \mathrm{C}$. Lower UTI was defined as a significant growth of bacteria with symptoms such as increased leakage or discomfort and a temperature $<$ $38.5^{\circ} \mathrm{C}$. ABU was defined as significant growth of bacteria without symptoms. All patients used prophylactic antibiotic therapy, usually sulfamethoxazole and trimethoprim, before CIC was introduced. The degree of incontinence in these patients before and during this therapy was also evaluated based on their parents' diaries.

\section{Chemicals and drugs}

Oxybutynin chloride and HPC were purchased from SigmaAldrich Co. (St. Louis, MO, USA). All other chemicals were of reagent grade.

\section{Results}

Follow-up durations were 118 months, 126 months, 127 months, and 51 months for the four patients. The CMG data are shown in Table 2, and typical traces are shown in Figure 1 (patient 1). Before treatment, all patients presented with low bladder compliance and detrusor overactivity. After 1 year, 
Table 2. Bladder Compliance, Detrusor Overactivity and Urinary Incontinence Before and After Treatment

\begin{tabular}{|c|c|c|c|c|c|c|c|c|c|c|c|c|}
\hline \multirow{2}{*}{ Patient } & \multicolumn{4}{|c|}{ Bladder compliance $\left(\mathrm{cm} \mathrm{H}_{2} \mathrm{O} / \mathrm{mL}\right)$} & \multicolumn{4}{|c|}{ Detrusor overactivity } & \multicolumn{4}{|c|}{ Urinary incontinence } \\
\hline & Before & 1 y later & 3 y later & 10 y later & Before & 1 y later & 3 y later & 10 y later & Before & 1 y later & 3 y later & 10 y later \\
\hline 1 & 1.7 & 6.7 & 6.5 & $3.7^{\mathrm{a}}$ & + & + & - & $+^{\mathrm{a}}$ & 3 times/m & None & None & 2.3 times $/ \mathrm{d}^{\mathrm{a}}$ \\
\hline 3 & 0.1 & 10.0 & 9.3 & 9.7 & + & - & - & - & None & None & None & None \\
\hline 4 & 2.5 & NP & 5.9 & 11.8 & + & NP & - & - & 4.5 times $/ \mathrm{d}$ & None & None & None \\
\hline
\end{tabular}

y: years; m: month; d: day; NP: not performed. aat 118 months.
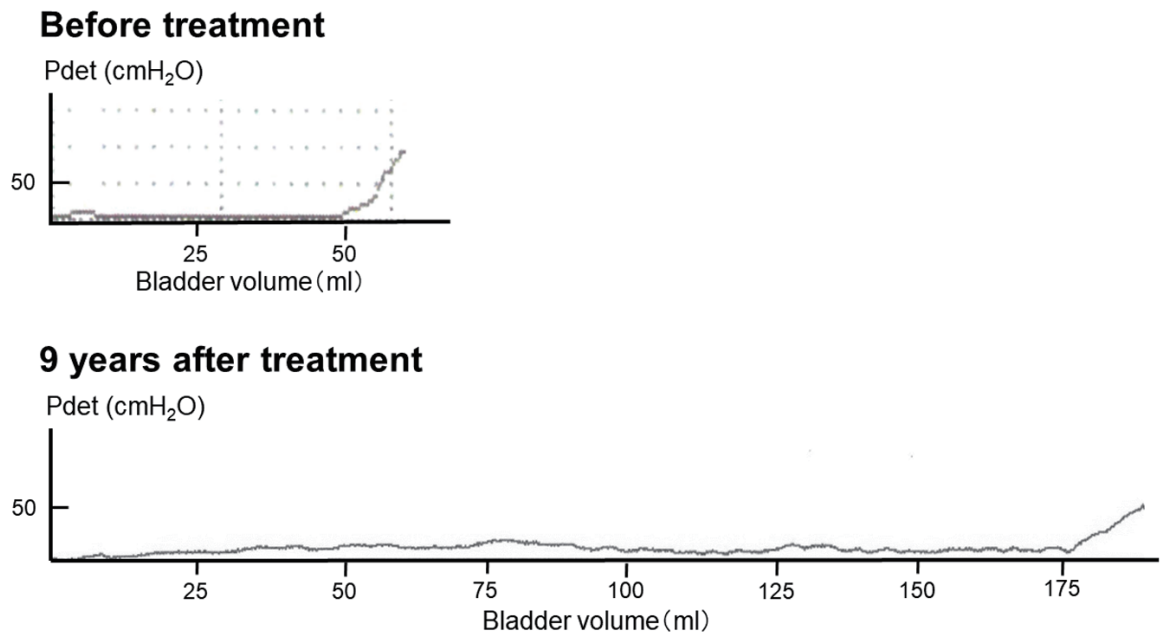

Figure 1. Cystometrograms of patient 1 before and after treatment.

bladder compliance improved in patients 1, 2, and 3. At 3 years, detrusor overactivity was not observed in all patients. Bladder compliance at 3 years and 10 years after initiation of therapy was similar for three patients, and they are continuing modified intravesical oxybutynin therapy. One patient discontinued therapy at 118 months due to worsening of bladder compliance and upper UTI. Table 2 shows the degree of incontinence before and during therapy. In patients 1,2 , and 4 , a significant improvement in the degree of incontinence was achieved. In the case of patient 3 , because she had suffered from urinary retention before therapy, no change in the degree of incontinence was observed.

No anticholinergic systemic AEs were observed in any of the patients. Patient 1 was able to increase the interval between catheterizations from $3 \mathrm{~h}$ before treatment to $6 \mathrm{~h}$ after treatment with no incontinence between catheterizations. In patient 3 , the indwelling urethral catheter could be replaced with CIC, due to improvement in bladder compliance ( $5 \mathrm{CICs} /$ day). The UTI status of all patients is summarized in Table 3. In all patients, the incidence of UTI did not increase during treatment. Two patients (patients 2 and 3) did not experience ABU for more than 3 years after instituting treatment.

\section{Discussion}

Although the number of patients in this study was small, all of them showed improved bladder compliance and a reduced

Table 3. Incidence of UTIs

\begin{tabular}{lllll} 
Patient & $\begin{array}{l}\mathbf{1} \text { before instituting } \\
\text { treatment }\end{array}$ & $\begin{array}{l}\mathbf{1} \text { y after instituting } \\
\text { treatment }\end{array}$ & $\begin{array}{l}\mathbf{3} \text { y after instituting } \\
\text { treatment }\end{array}$ & $\begin{array}{l}\mathbf{1 0} \text { y after instituting } \\
\text { treatment }\end{array}$ \\
\hline 1 & Lower UTI (3 times) & None & None & Upper UTI (2 times) \\
2 & ABU & ABU & None & None \\
3 & ABU & None & None & None \\
4 & ABU & ABU & ABU & ABU \\
\hline
\end{tabular}

UTI: urinary tract infection; $\mathrm{ABU}$ : asymptomatic bacteriuria. ${ }^{\text {aat }} 118$ months. 
degree of urinary incontinence. The present data suggest that modified intravesical oxybutynin is an effective treatment option for children with neurogenic bladder. To the best of our knowledge, this study is the first to assess the efficacy of 10year use of modified intravesical oxybutynin for children with this disorder.

The aim of treatment for patients who have neurogenic bladder due to spinal cord disease is to protect renal function, reduce urinary infections, ameliorate urinary incontinence, and increase quality of life [9]. The primary goals of pediatric neurogenic bladder management are to normalize vesical pressure and achieve social continence [4]. While it is true that oral antimuscarinic pharmacotherapy is the first-line therapy, the high incidence of systemic AEs has compromised compliance, resulting in dose reductions or even discontinuation of treatment.

In 1989, Brendler et al first reported treatment with intravesical oxybutynin chloride for neurogenic bladder [10]. Since then, evidence of the efficacy of intravesical oxybutynin has been reported $[1,2,11,12]$. Generally, the majority of children with neurogenic bladder (70-90\%) respond well to oral antimuscarinic agents with or without CIC [13]. This therapeutic approach allows a high concentration of the drug to be delivered to the target tissue with the maximal pharmacological response and, theoretically, minimal AEs. However, the pharmacokinetic mechanisms of oxybutynin are not well known. It has been suggested that intravesical oxybutynin chloride has a longer half-life, even with the higher peak levels. In fact, Madersbacher and Knoll reported that the reabsorption of intravesical oxybutynin is slower than that after an oral dose; peak levels are reached later and are maintained at a higher level after $6 \mathrm{~h}$ [12]. An increasing amount of evidence suggests that high plasma levels of N-desethyl-oxybutynin (DEOB), an active oxybutynin metabolite, cause systemic AEs $[1,2]$. Indeed, Lehtoranta et al reported that a significantly lower ratio of the area under the plasma concentration time curve (AUC) of DEOB over oxybutynin may reduce systemic AEs [2]. Therefore, intravesical oxybutynin chloride, which reduces plasma levels of DEOB to lower than that with oral administration of oxybutynin chloride, is an effective therapy for overactive bladder, which is intolerable or with severe AEs that occur after oral oxybutynin medication. The mechanism of action of intravesical oxybutynin may involve a direct effect on the bladder muscle, a topical anesthetic effect, or an indirect effect of absorbed oxybutynin and its metabolites [14]. Di Stasi et al also reported that this therapeutic effect is a direct localized action within the bladder wall, probably through local anesthesia of the afferent arm of a reflex arc and possibly via a small degree of diffusion down to the M3 receptors in the detrusor [15]. Immunohistochemistry and reverse transcription polymerase chain reaction (RT-PCR) techniques have shown that both M2 and M3 muscarinic receptor subtypes are expressed in the rat urothelium, and that M2 receptors are expressed on the human urothelium and are closely associated with sensory nerves [16]. Recently, Kim et al reported that intravesical administration of oxybutynin not only suppresses muscarinic receptor-mediated detrusor muscle contraction, but also blocks muscarinic receptors in bladderafferent pathways in rats [17]. Actually, Haga et al reported that oral administration of low-dose oxybutynin decreased cfos expression in the spinal cord, which was induced by continuous infusion of saline into the rat bladder [18]. Further studies are needed to resolve the pharmacokinetics of intravesical oxybutynin chloride.

Intravesical oxybutynin chloride is an effective method with fewer AEs than oral medication [9]. However, the duration of this method often does not last long enough. Weese et al reported that, of 42 patients administered intravesical oxybutynin medication, nine had difficulty retaining the solution in the urinary bladder [19]. Based on these and other previous reports, difficulty retaining the solution in the bladder is an important problem. To reduce the above-mentioned transient effects of intravesical oxybutynin chloride, some clinical techniques have been reported. HPC is an agent that adheres to bladder mucosa and retains drugs in the bladder longer. Mizunaga et al reported that they developed a new oxybutynin solution containing HPC, a mucosal adhesive substance. They reported that plasma concentrations of oxybutynin after intravesical HPC-modified oxybutynin were significantly lower than that after instillation of oxybutynin alone, and the effect of HPC-modified oxybutynin lasted longer than that of oxybutynin alone. Moreover, favorable clinical effects were achieved, and no AEs were observed for more than 6 months during medication periods [20]. It has been suggested that modification with HPC may reduce the absorption of oxybutynin from bladder mucosa, so that oxybutynin is retained in the bladder for longer, and it may reduce anticholinergic AEs compared to those of intravesical oxybutynin without HPC. In the present study, muscarinic AEs were not seen in any of the patients. Recent study demonstrated that intravesical application of $0.1 \%$ oxybutynin hydrochloride solution (30 mg oxybutynin hydrochloride/day) was equal to oral administration in adult patients with neurogenic bladder [1]. On the other hand, the present data suggest that intravesical oxybutynin hydrochloride (2.5 mg oxybutynin hydrochloride/day) with HPC is an effective and relatively safe long-term therapeutic option for children with neurogenic bladder. Therefore, modified intravesical oxybutynin therapy may be effective even in a small dose of oxybutynin hydrochloride.

\section{Limitations}

There are several limitations to this study. First, in all retrospective analyses, there are inherent limitations. Second, the pooled sample size was not powered to demonstrate the safety or efficacy of long-term modified intravesical oxybutynin therapy in children with neurogenic bladder. Third, the ideal study would be a prospective, randomized, placebo controlled trial. Challenges and limitations of trial design for pediatric patients with refractory neurogenic bladder include patient heterogeneity, and the small number of patients in whom traditional urotherapy fails. However, the long-term effects of modified intravesical oxybutynin therapy were confirmed by urodynamic studies. Therefore, we believe that, although patient numbers were limited, modified intravesical oxybutynin therapy is very valuable for patients who do not respond to oral antimuscarinics. 


\section{Conclusions}

Based on the data from this study, although patient numbers were limited, long-term use of modified intravesical oxybutynin therapy in children with neurogenic bladder with detrusor overactivity and/or high intravesical pressure during the filling phase could be an important option for improving continence and bladder compliance.

\section{Competing Interest}

The authors declare that they have no competing interest.

\section{Grant Support}

None.

\section{Financial Disclosure}

None.

\section{References}

1. Schroder A, Albrecht U, Schnitker J, Reitz A, Stein R. Efficacy, safety, and tolerability of intravesically administered $0.1 \%$ oxybutynin hydrochloride solution in adult patients with neurogenic bladder: A randomized, prospective, controlled multi-center trial. Neurourol Urodyn. 2016;35(5):582-588.

2. Lehtoranta K, Tainio H, Lukkari-Lax E, Hakonen T, Tammela TL. Pharmacokinetics, efficacy, and safety of intravesical formulation of oxybutynin in patients with detrusor overactivity. Scand J Urol Nephrol. 2002;36(1):18-24.

3. Guerra LA, Moher D, Sampson M, Barrowman N, Pike J, Leonard M. Intravesical oxybutynin for children with poorly compliant neurogenic bladder: a systematic review. J Urol. 2008;180(3):1091-1097.

4. Lazarus J. Intravesical oxybutynin in the pediatric neurogenic bladder. Nat Rev Urol. 2009;6(12):671-674.

5. Saito M, Watanabe T, Tabuchi F, Otsubo K, Satoh K, Miyagawa I. Urodynamic effects and safety of modified intravesical oxybutynin chloride in patients with neurogenic detrusor overactivity: 3 years experience. Int J Urol. 2004;11(8):592-596.

6. Hayashi A, Saito M, Okada S, Hanada T, Watanabe T, Satoh K, Kanzaki S. Treatment with modified intravesical oxybutynin chloride for neurogenic bladder in children. J Pediatr Urol. 2007;3(6):438-442.

7. Kasabian NG, Vlachiotis JD, Lais A, Klumpp B, Kelly
MD, Siroky MB, Bauer SB. The use of intravesical oxybutynin chloride in patients with detrusor hypertonicity and detrusor hyperreflexia. J Urol. 1994;151(4):944-945.

8. Palmer LS, Zebold K, Firlit CF, Kaplan WE. Complications of intravesical oxybutynin chloride therapy in the pediatric myelomeningocele population. J Urol. 1997;157(2):638-640.

9. Inoue S, Saito M, Honda M, Dimitriadis F, Takenaka A. Intravesical oxybutynin for neurogenic bladder in children. Pediat Therapeut. 2012;2:138.

10. Brendler CB, Radebaugh LC, Mohler JL. Topical oxybutynin chloride for relaxation of dysfunctional bladders. J Urol. 1989;141(6):1350-1352.

11. Madersbacher H, Jilg G. Control of detrusor hyperreflexia by the intravesical instillation of oxybutynin hydrochloride. Paraplegia. 1991;29(2):84-90.

12. Madersbacher H, Knoll M. Intravesical application of oxybutynine: mode of action in controlling detrusor hyperreflexia. Preliminary results. Eur Urol. 1995;28(4):340344.

13. Amend B, Hennenlotter J, Schafer T, Horstmann M, Stenzl A, Sievert KD. Effective treatment of neurogenic detrusor dysfunction by combined high-dosed antimuscarinics without increased side-effects. Eur Urol. 2008;53(5):1021-1028.

14. Reitz A, Schurch B. Intravesical therapy options for neurogenic detrusor overactivity. Spinal Cord. 2004;42(5):267272.

15. Di Stasi SM, Giannantoni A, Navarra P, Capelli G, Storti L, Porena M, Stephen RL. Intravesical oxybutynin: mode of action assessed by passive diffusion and electromotive administration with pharmacokinetics of oxybutynin and N-desethyl oxybutynin. J Urol. 2001;166(6):2232-2236.

16. Saito M, Kinoshita Y, Satoh I, Shinbori C, Suzuki H, Yamada M, Watanabe T, et al. Ability of cyclohexenonic long-chain fatty alcohol to reverse diabetes-induced cystopathy in the rat. Eur Urol. 2007;51(2):479-487; discussion 487-478.

17. Kim Y, Yoshimura N, Masuda H, de Miguel F, Chancellor MB. Antimuscarinic agents exhibit local inhibitory effects on muscarinic receptors in bladder-afferent pathways. Urology. 2005;65(2):238-242.

18. Haga N, Aikawa K, Shishido K, Takahashi N, Yanagida T, Yamaguchi O. Effect of long-term oxybutynin administration on c-Fos expression in spinal neurons: inhibition of antimuscarinics on bladder afferents in conscious rats. Urology. 2009;73(1):200-204.

19. Weese DL, Roskamp DA, Leach GE, Zimmern PE. Intravesical oxybutynin chloride: experience with 42 patients. Urology. 1993;41(6):527-530.

20. Mizunaga M, Miyata M, Kaneko S, Yachiku S, Chiba K. Intravesical instillation of oxybutynin hydrochloride therapy for patients with a neuropathic bladder. Paraplegia. 1994;32(1):25-29. 